\title{
EGF and HB-EGF enhance the proliferation of programmable cells of monocytic origin (PCMO) through activation of MEK/ERK signaling and improve differentiation of PCMO-derived hepatocyte-like cells
}

Ayman Hyder ${ }^{1}$, Sabrina Ehnert ${ }^{2}$, Hebke Hinz ${ }^{1}$, Andreas K Nüssler ${ }^{2}$, Fred Fändrich ${ }^{1}$ and Hendrik Ungefroren ${ }^{1,3^{*}}$

\begin{abstract}
Background: Hepatocyte-like cells (NeoHepatocytes) generated from a peripheral blood monocyte-derived stem cell-like cell (the PCMO) are a promising alternative for primary hepatocytes in cell transplantation studies to cure liver diseases. However, to be therapeutically effective NeoHepatocytes are needed in large quantities. It was the aim of the present study to investigate i) whether the proportion of actively proliferating NeoHepatocytes can be enhanced by supplementing the PCMO differentiation medium (containing M-CSF, IL-3, and human serum) with either EGF or HB-EGF and ii) which signaling pathway underlies the promitotic effect.

Results: EGF and HB-EGF enhanced cell proliferation of PCMOs as demonstrated by increased expression of cycle control genes (ABL, ANAPC2, CDC2, CDK4, CDK6), phosphorylation of the retinoblastoma protein, and increased PCMO cell numbers after stimulation with EGF or HB-EGF. EGF also raised the number of monocytes expressing the proliferation marker Ki67. PCMOs expressed the EGF receptors EGFR (ERBB1) and ERBB3, and expression of both increased during PCMO generation. Phosphoimmunoblotting of PCMOs indicated that both EGF and HB-EGF activated MEK-1/2 and ERK1/2 in a concentration-dependent fashion with the effect of EGF being more prominent. EGF treatment further decreased expression of $\mathrm{p} 47^{\text {phox }}$ and increased that of Nanog indicating enhanced dedifferentiation and pluripotency, respectively. Treatment with both EGF and HB-EGF resulted in NeoHepatocytes with improved functional parameters.

Conclusions: The results suggested that the addition of EGF or HB-EGF to PCMO differentiation medium superactivates MEK/ERK signaling which then increases both PCMO proliferation, number, and functional differentiation of PCMO-derived NeoHepatocytes.
\end{abstract}

Keywords: EGF, Hepatocyte-like cells, MEK, Extracellular signal-regulated kinase, Proliferation, Programmable cells of monocytic origin

\footnotetext{
* Correspondence: hendrik.ungefroren@uksh.de

'Clinic for Applied Cellular Medicine, UKSH, Campus Kiel, Arnold-Heller

Strasse 3, Hs. 18, 24105 Kiel, Germany

${ }^{3}$ First Department of Medicine, UKSH, Campus Lübeck, Ratzeburger Allee 160,

23538 Lübeck, Germany

Full list of author information is available at the end of the article
} 


\section{Background}

Although hepatocyte transplantation is a therapeutic option for end-stage liver diseases, cell material is scarce due to a critical shortage of liver tissues and the lack of protocols that allow maintaining the differentiated hepatocyte phenotype in culture for more than a week. Thus, generation of hepatocyte-like cells from stem cells or stem cell-like cells may represent a promising alternative [1]. One such cell type with inherent stem cell-like features is the human peripheral blood monocyte [2-5]. By initially inducing a process of dedifferentiation we have generated from these cells a more plastic derivative termed "programmable cell of monocytic origin" (PCMO). PCMOs are prone to acquire functional activities of hepatocyte-like cells (NeoHepatocytes) upon stimulation with appropriate differentiation media in vitro [2,3], and in vivo following transplantation into mice [2].

From the clinical point of view, a major obstacle in cell transplantation is the large amount of cells required to achieve a therapeutic effect in patients. Despite an already large number of cells that can be retrieved from blood products the overall numbers of NeoHepatocytes obtained after the two-step dedifferentiationdifferentiation protocol are still low and insufficient. One possibility to increase NeoHepatocyte cell numbers is by inducing the cells to proliferate. This is more likely to be possible at or before the PCMO stage as the NeoHepatocyte differentiation from PCMO is mutually exclusive with proliferation. Indeed, during conversion of peripheral blood monocytes into PCMOs, a process involving dedifferentiation, a fraction of monocytes resume proliferation in vitro in response to macrophage-colony stimulating factor (M-CSF), interleukin-3 (IL-3), and human serum [2]. The extent of proliferation however, was not sufficient to substantially increase the overall cellular yield of NeoHepatocytes. If the rate of proliferation and/or the percentage of mitotically active monocytes could be enhanced prior to induction of differentiation, then an increased number of NeoHepatocytes may be obtained, thereby increasing the chance for successful NeoHepatocyte transplantations. Ideally, a modification of the PCMO generation procedure, e.g. by addition of growth-stimulatory factor(s), should not only enhance mitotic activity but also the plasticity of PCMOs in such a way that the resulting NeoHepatocytes become more hepatocyte-like [6]. Interestingly, a subpopulation of human monocytes that proliferates in vitro in response to M-CSF has been suspected to be less mature and hence more stem cell-like than other monocytes [7]. Therefore, the identification of growth factor signaling pathways that regulate proliferation of human monocytes may enhance both the quantity and quality of PCMO-derived NeoHepatocytes.
Epidermal growth factor (EGF) is known to induce proliferation in many kinds of cells [8-11] and its receptor is over-expressed in proliferative cells [12]. Another member from the EGF family, the 20-22 kDa glycoprotein Heparin-binding epidermal growth factor (HB-EGF) [13] was also reported to be a potent mitogen for many cell types [14-16]. Human peripheral blood monocytes were shown recently to express a functional EGF receptor (EGFR) $[17,18]$, while the EGF receptors c-ERBB2, 3 and 4 have not been studied. However, a link between EGF or HB-EGF and proliferation in monocytes has never been investigated. Analysis of the mechanism of receptor tyrosine kinase activation in monocytes may identify soluble factors that control PCMO self renewal. The present study aimed to investigate the expression and the activity of the epidermal growth factor receptor (ERBB) family in human peripheral monocytes and the role of EGF and HB-EGF on the outcome of PCMO generation and the subsequent differentiation into NeoHepatocytes.

\section{Results}

\section{Gene expression of EGF receptor family members in PCMOs}

We first sought to determine which EGF receptors are expressed in monocytes. For this purpose, RNA was isolated from monocyte cultures and processed for $\mathrm{qPCR}$ using primers for EGFR (also termed ERBB1), ERBB2, ERBB3, and ERBB4 as listed in Table 1. RT-PCR analysis of the four EGF receptors yielded a strong signal for EGFR and a weaker one for ERBB3 (Figure 1A). Since monocytes may be "contaminated" with lymphocytes, a negative control sample of highly purified lymphocytes was analyzed in parallel and shown to lack expression of both EGFR and ERBB3 (data not shown). This indicated that the amplification products for EGFR and ERBB3 were specifically derived from monocytes. Since the expression levels of some genes may differ during the development of PCMOs in culture, we isolated RNA from the developing PCMOs at different days of culture. The qPCR of these samples indicated that expression of both EGFR and ERBB3 initially increased during PCMO generation reaching a peak on the second day (ERBB3) and on the fourth day (EGFR) of culture and decreased thereafter (Figure 1B).

\section{EGF promotes proliferation during PCMO production}

Next, we examined the effect of EGF and HB-EGF on the proliferation of PCMOs (Figure 2). For this purpose, cells were cultured for 4 days in PCMO medium containing EGF or HB-EGF at different concentrations. Cells were prepared for immunofluorescence using Ki67 antibody as a proliferation marker and CD14 as a monocyte marker. The results showed a higher number of 
Table 1 Sequences of PCR primers

\begin{tabular}{|c|c|c|c|c|}
\hline Gene & Accession No. & Sense primer & Antisense primer & Product size (bp) \\
\hline EGFR & NM_005228.3 & ATGCTCTACAACCCCACCAC & GCCCTTCGCACTTCTTACAC & 193 \\
\hline c-erbB2 & NM_004448.2 & СССТСАТССАССАТААСАСС & GCCTGGCATTCACATACTCC & 279 \\
\hline c-erbB3 & NM_001982.3 & TACTTGGAACGGGGTGAGAG & ACTCTGCCGTCCACTCTTGT & 219 \\
\hline c-erbB4 & NM_005235.2 & AGTCAGTGTGTGCAGGAACG & CTCCAGAGGCAGGTAACGAA & 224 \\
\hline Nanog & NM_024865 & GATTTGTGGGCCTGAAGAAAACT & AGGAGAGACAGTCTCCGTGTGAG & 79 \\
\hline GAPDH & NM_002046 & TTGCCATCAATGACCCCTTCA & CGCCCCACTTGATTTTGGA & 174 \\
\hline$\beta$-actin & NM_031144 & GATATCGCTGCGCTCGTC & TCCATATCGTCCCAGTTGG & 239 \\
\hline ANAPC2 & NM_013366.3 & CCAGTACAGGCGGTGATCTT & GCTCTCGTCGTCACTGTCAA & 228 \\
\hline$A B L-1$ & NM_005157.4 & AACACCCTAACCTGGTGCAG & CAAGTGGTTCTCCCCTACCA & 248 \\
\hline CDC2 (CDK1) & NM_001786.4 & GGGGTCAGCTCGTTACTCAA & GATGCTAGGCTTCCTGGTTTC & 225 \\
\hline CDK4 & NM_000075.2 & CTGACCGGGAGATCAAGGTA & AGCCAGCTTGACTGTTCCAC & 224 \\
\hline CDK6 & NM_001145306.1 & TCCCAGGAGAAGAAGACTGG & GGTCCTGGAAGTATGGGTGA & 198 \\
\hline
\end{tabular}

Ki67/CD14 double-positive cells in both EGF and HBEGF-treated cultures (Figure 2a). However, quantification of these cells showed that the HB-EGF but not the EGF effect closely missed statistical significance (Figure 2B). No statistically significant differences of Ki67/CD14-positive cell counts were observed among

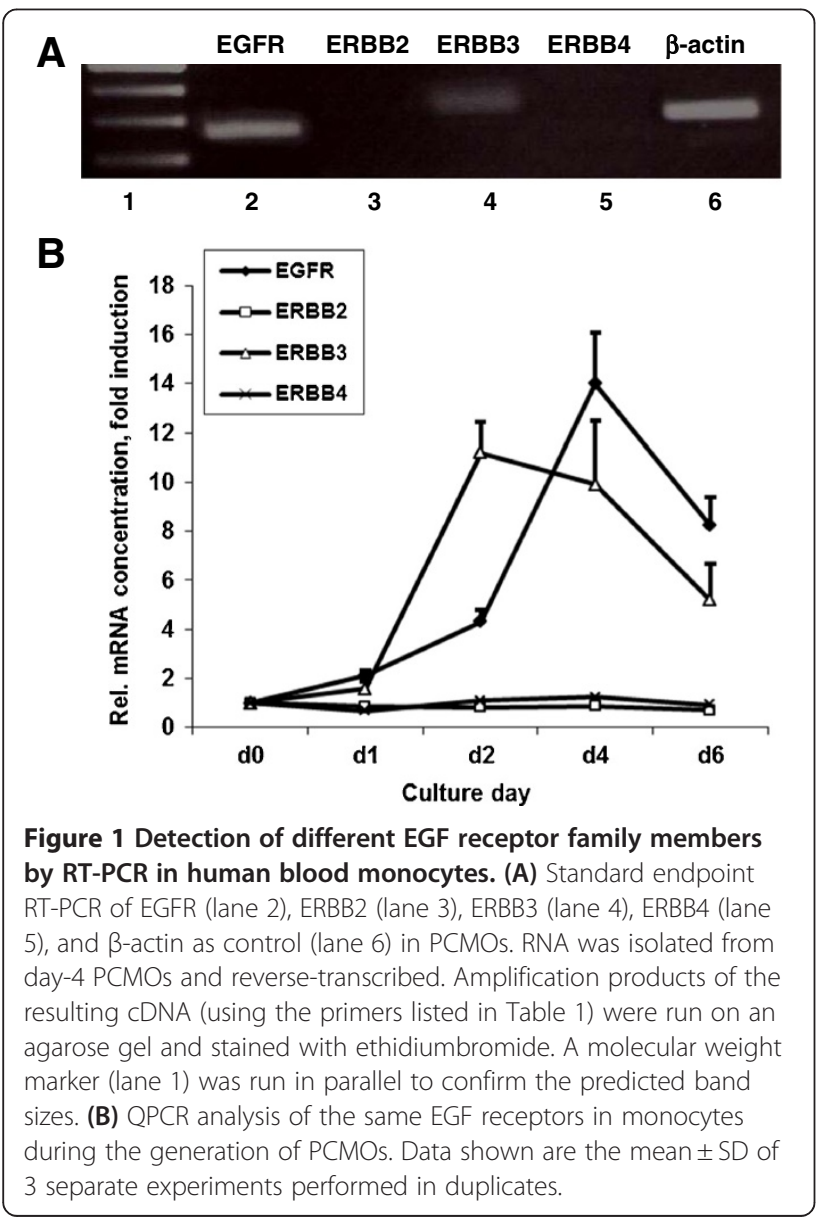

different concentrations of the same treatment. These data clearly show that the addition of EGF enhanced the proliferative activity of monocytes in PCMO generation medium. EGF-induced proliferation temporally correlated with cell cycle activation. In order to investigate whether EGF-induced proliferation was associated with the expression of specific cell-cycle regulatory genes, we treated monocytes with different concentrations of EGF or HB-EGF and performed qPCR analysis as described in the Methods section. As seen in Table 2, both EGF and HB-EGF up-regulated the expression of ABL, ANAPC2, CDC2, CDK4, and CDK6, each of which is involved in different stages of the cell cycle.

RNA was isolated from PCMOs after 4-day culture with or without EGF or HB-EGF (10, 50, $100 \mu \mathrm{g} / \mathrm{L})$ and transcribed to cDNA. QPCR was applied using primer pairs listed in Table 1. Data are presented as mean \pm SEM of $\mathrm{N}=4$ and represent the fold-change in comparison with control PCMOs (cultured without HB-EGF or EGF), the values of which were considered as 1 . Statistical analysis: $\mathrm{a}=$ significantly different from the control, b: significantly different from the corresponding HB-EGF value.

The retinoblastoma protein $(\mathrm{pRb})$ plays a pivotal role in the negative control of the cell cycle and prevents the cell from replicating damaged DNA by blocking progression through G1 into S phase. Its inhibitory role on cell cycle progression is carried out in the hypophosphorylated state, while phosphorylation inactivates pRb [19]. We have analysed the phosphorylation state of $\mathrm{pRb}$ in PCMOs generated in the presence of either EGF or HBEGF (Figure 2C). The results show that treatment with HB-EGF increased the phosphorylation of $\mathrm{pRb}$, while EGF caused its hyperphosphorylation. In control cells, however, only the active non-phosphorylated form was present (Figure 2C).

We have also investigated cyclin A protein in the same samples. Cyclin A defines control points of the cell cycle. 


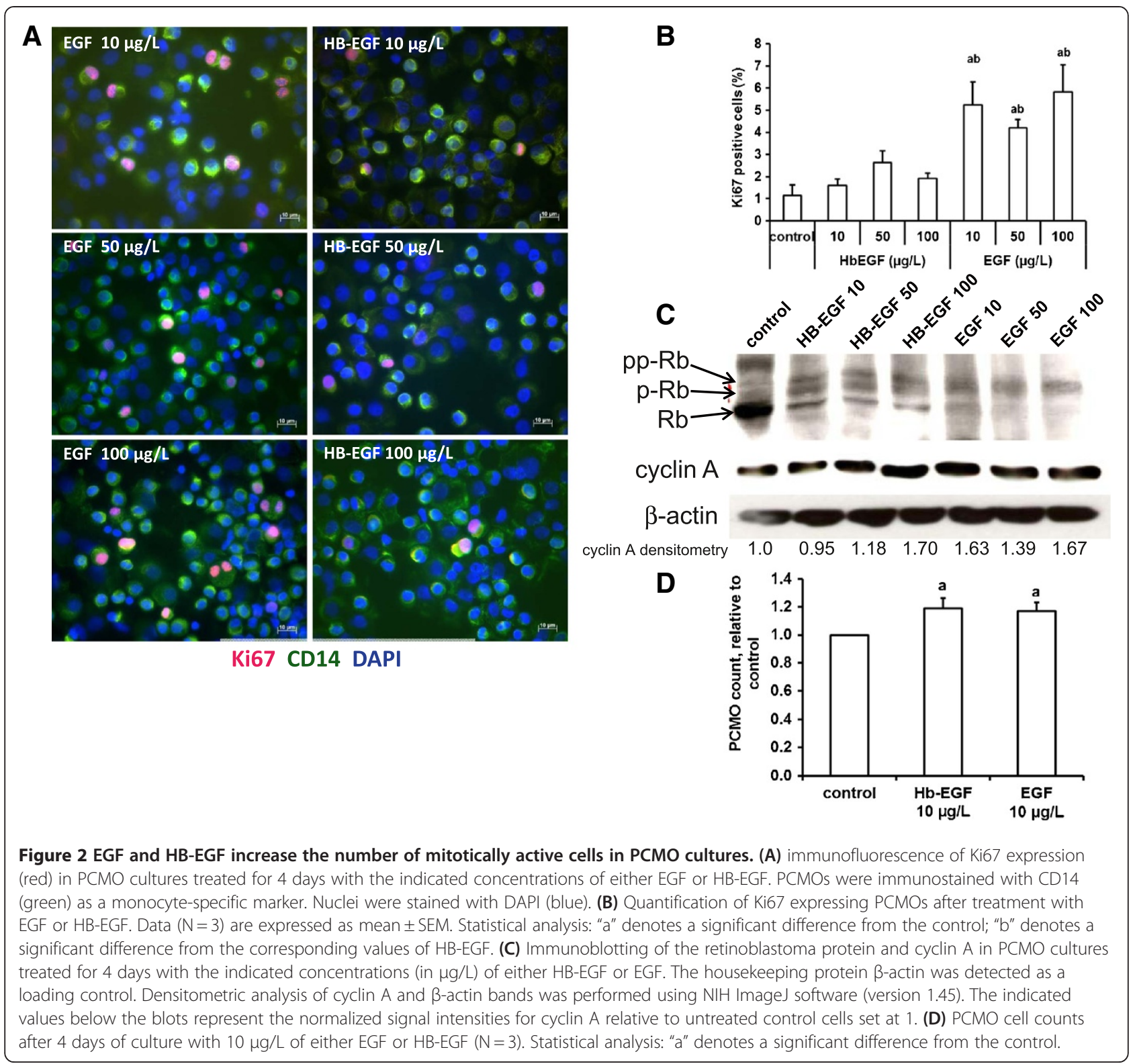

It binds both CDK2 and CDC2 giving rise to two distinct cyclin A kinase activities, one appearing in $\mathrm{S}$ phase and the other one in G2 phase [20]. Immunoblotting indicated an increase in cyclin A expression upon treatment of PCMOs with 50 and $100 \mu \mathrm{g} / \mathrm{L}$ HB-EGF and with all three concentrations of EGF (Figure 2C).

Finally, we performed cell counting of PCMOs cultured for 4 days with either $10 \mu \mathrm{g} / \mathrm{L}$ EGF or HB-EGF

Table 2 Expression of cell cycle genes during the development of PCMOs cultured in the presence of HB-EGF or EGF

\begin{tabular}{llllll}
\hline & ABL & ANAPC2 & CDC2 & CDK4 & CDK6 \\
\hline Hb-EGF 10 & $1.40 \pm 0.19^{\mathbf{a}}$ & $1.74 \pm 0.62$ & $1.88 \pm 0.74$ & $1.53 \pm 0.33$ & $2.42 \pm 1.10$ \\
Hb-EGF 50 & $1.26 \pm 0.15^{\mathbf{a}}$ & $1.40 \pm 0.45$ & $1.81 \pm 0.77$ & $1.56 \pm 0.33$ & $2.09 \pm 0.80$ \\
Hb-EGF 100 & $1.48 \pm 0.43$ & $1.63 \pm 0.66$ & $2.23 \pm 0.95$ & $1.47 \pm 0.27$ & $2.35 \pm 1.06$ \\
EGF 10 & $2.24 \pm 0.18^{\mathbf{a b}}$ & $1.78 \pm 0.43$ & $3.01 \pm 1.05^{\mathbf{a b}}$ & $1.57 \pm 0.17$ & $2.47 \pm 0.96$ \\
EGF 50 & $1.69 \pm 0.20^{\mathbf{a b}}$ & $1.70 \pm 0.42$ & $1.99 \pm 0.42^{\mathbf{a}}$ & $1.39 \pm 0.10$ & $2.15 \pm 0.87$ \\
EGF 100 & $1.74 \pm 0.30^{\mathbf{a b}}$ & $1.60 \pm 0.38$ & $2.02 \pm 0.50^{\mathbf{a}}$ & $1.53 \pm 0.14$ & $2.38 \pm 0.93$ \\
ANOVA $=$ & $\mathbf{0 . 0 0 3 3}$ & $\mathbf{0 . 0 7 4 8}$ & $\mathbf{0 . 0 3 6}$ & $\mathbf{0 . 2 6 1}$ & $\mathbf{0 . 0 7 5 6}$ \\
\hline
\end{tabular}


(Figure 2D). The results demonstrated a moderate but significant increase over the control in total cell counts after both treatments. No difference was observed between the two treatments. Together, the data show that EGF and HB-EGF are suitable tools to expand the total cell number of PCMOs and that this largely occurs through an increase in the mitotic/cell cycle activity of monocytes.

\section{EGF treatment attenuates expression of $\mathrm{p} 47^{\text {phox }}$ and enhances expression of Nanog in PCMOs}

During the generation of PCMOs, monocytes downregulate markers of differentiation, e.g. $\mathrm{p} 47^{\mathrm{phox}}$ an essential subunit of the reactive oxygen producing enzyme NAD (P)H oxidase and upregulate markers of pluripotency, e.g. Nanog [6]. We have examined the effect of EGF and HB-EGF on the expression of $\mathrm{p} 47^{\text {phox }}$ by immunoblotting (Figure 3A) and on the expression of Nanog by $\mathrm{qPCR}$ (Figure 3B). The $\mathrm{p} 47^{\mathrm{phox}}$ protein levels were clearly lower on day 4 of culture which was particularly prominent in EGF-treated cultures (Figure 3A). No

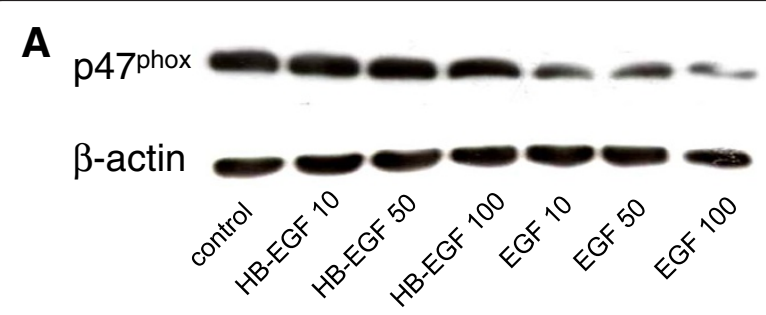

B

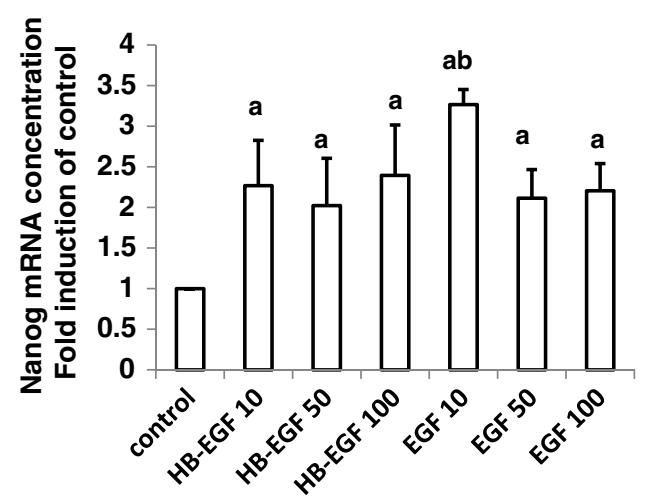

Figure 3 Regulation of Nanog and $p 47^{\text {phox }}$ expression by EGF or HB-EGF in PCMOs. (A) Measurement of Nanog expression by QPCR in PCMOs treated for 4 days with the indicated concentrations (in $\mu \mathrm{g} / \mathrm{L}$ ) of HB-EGF or EGF. Data (mean $\pm \mathrm{SEM}, \mathrm{N}=4$ ) were normalized to the expression level of GAPDH. ANOVA: $p<0.01$; $a=$ all values of EGF and HB-EGF are significantly higher than that of the control; $b=$ significant difference between EGF and the corresponding HB-EGF value. (B) Immunoblot analysis of $\mathrm{p} 47^{\text {phox }}$ in day-4 PCMOs treated with either HB-EGF or EGF at the indicated concentrations. The $\beta$-actin protein was detected as a loading control. differences were observed between treatments with different concentrations of EGF. Both EGF and HB-EGF caused a more than 2-fold increase in the mRNA levels of Nanog (Figure 3B). Statistically significant differences were observed neither among EGF and HB-EGF treatments nor among different concentrations of each growth factor. The data suggest that EGF can enhance both the extent of dedifferentiation and pluripotency.

\section{MEK/ERK signaling drives proliferation in PCMOs and is superactivated by EGF and HB-EGF}

ERK and MEK activation is involved in M-CSF and EGF-induced proliferation of PCMOs. We have previously shown that during PCMO culture, a subset of monocytes resumes proliferation. To test whether this is associated with activation of MEK/ERK signaling, we performed immunoblot analysis of ERK activation (Figure 4A). ERK phosphorylation during PCMO generation peaked on day 3-4 of culture and this increase coincided with peak mitotic activity [2]. This suggested that ERK activation is causally involved in driving proliferation of monocytes/PCMOs. To test this more directly, we inhibited MEK1 with U0126 during PCMO culture and assessed the number of cells on day 6. The total number of cells was low, indicating that MEK/ERK signaling is crucial for PCMO proliferation (Figure 4B).

Since both EGF and HB-EGF are known to stimulate ERK activation, we reasoned that these agents may enhance proliferation by superactivating the MEK/ERK pathway. To test this prediction, PCMOs were generated in standard PCMO differentiation medium in the absence or presence of either EGF or HB-EGF and subjected to immunoblot analysis of phospho-MEK and phospho-ERK. The results indicated that both EGF and HB-EGF activated MEK and ERK and that the effect was concentration-dependent and more prominent in EGFtreated than in HB-EGF-treated PCMOs (Figure 4C).

\section{Effect of EGF and HB-EGF on NeoHepatocyte function} Ideally, a modification of the PCMO generation procedure should not only enhance proliferation but also the stem cell features of PCMOs in a way that the resulting NeoHepatocytes become more hepatocyte-like. We therefore tested whether adding EGF and HB-EGF to the PCMO generation medium would alter functional parameters of the Neoepatocytes. Control PCMOs and PCMOs generated in the presence of either EGF or HBEGF were allowed to differentiate into NeoHepatocytes for 2 weeks and at the end of this period were analysed for hepatocyte-specific functions (Figure 5).

NeoHepatocytes, regardless of treatment, including the control, formed and secreted urea in similar amounts as under basic conditions. Addition of $\mathrm{NH}_{4} \mathrm{Cl}$ increased urea formation in all settings. However, it was 
A

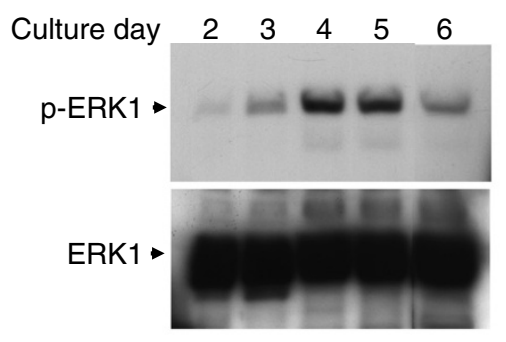

B

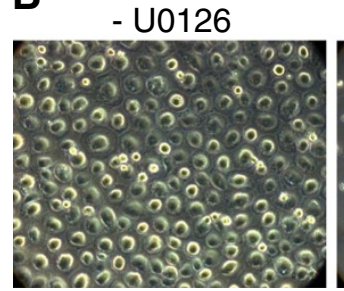

+ U0126

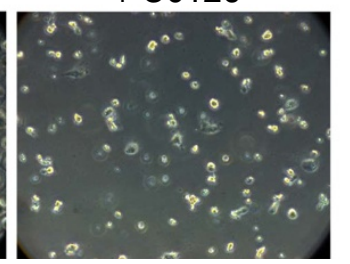

C

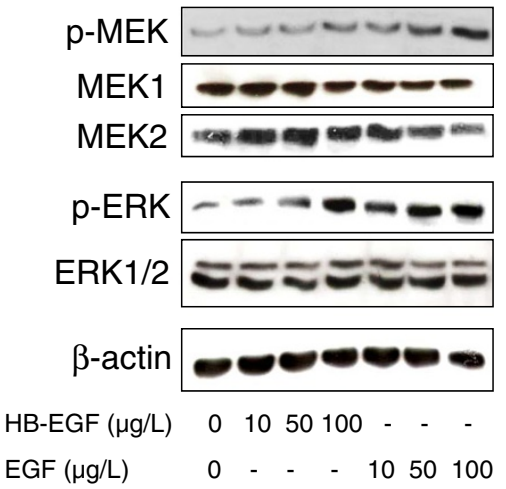

Figure 4 MEK/ERK signaling drives proliferation in PCMOs and is superactivated by EGF and HB-EGF. (A) M-CSF and II-3 induce phosphorylation of ERK ( $p E R K$ ). Protein samples were obtained from PCMOs cultured for various days (as indicated) in standard PCMO differentiation medium. (B) Inhibition of ERK was sufficient to inhibit PCMO proliferation. Cells were cultured with either solvent (left) or the ERK inhibitor U0126 (right) for 6 days and images were taken. Original magnification: 50x. (C) HB-EGF and EGF induce phosphorylation of MEK and ERK. Phospho-MEK (p-MEK), MEK1/2, phospho-ERK (p-ERK), and ERK1/2 expression was examined by immunoblotting in lysates of day-4 PCMOs that have been treated with the indicated concentrations of either HB-EGF or EGF.

higher in NeoHepatocytes obtained from PCMOs generated in the presence of HB-EGF. NeoHepatocytes regardless of treatment, including the control, all secreted glucose at similar rates. To measure the ability of the cells to perform gluconeogenesis, the Na-pyruvatecontaining incubation buffer was supplemented with $\mathrm{Na}$-L-lactate. Stimulation with pyruvate/lactate induced higher glucose secretion compared to non-stimulated cultures. As for urea, the effect was higher in NeoHepatocytes obtained from PCMOs generated in the presence of HB-EGF.

NeoHepatocytes exhibit phase I and II enzyme activities. However, levels were significantly lower compared to primary human hepatocytes [21] and could be enhanced by replacing the FCS with autologous serum [21]. We investigated the effect of EGF and HB-EGF on the activity of three different cytochrome $\mathrm{P}_{450}$ isoforms $(1 \mathrm{~A} 1 / 2,2 \mathrm{D} 6$, and 3A4) and a phase II enzyme (UDPglucuronosyl-transferase). The activities measured in cells varied between the different treatments. CYP1A1/2 activity was similar in, NeoHepatocytes obtained from PCMOs treated with either EGF or HB-EGF, and the effect of both was concentration-dependent. CYP2D6 activity was higher in NeoHepatocytes obtained from PCMOs treated with HB-EGF than those treated with EGF. This situation was reversed for the activity of CYP3A4. The activity of the phase II enzyme UDPglucuronosyl-transferase was similar for both treatments, but higher than that of the control.

\section{Discussion}

Peripheral blood monocytes can be reprogrammed to generate a kind of stem cell-like cell (PCMO), which is sensitive to differentiation into hepatocyte-like cells $[2,3]$. In view of a potential clinical use of these cells in regenerative cell therapies such as treatment of endstage liver diseases, the identification of factors capable of increasing the expansion of PCMOs/NeoHepatocytes is of great importance.

M-CSF and IL-3 present in the PCMO generation medium induce a proliferative response in a subset of monocytes through activation of MEK/ERK1/2 signaling (see Figure 4). Since this signaling pathway is also activated by EGF and HB-EGF and their receptors and is involved in the proliferation of many cell types [14-16], we reasoned that EGF should be able to further stimulate PCMO proliferation. In agreement with this assumption, we detected the expression of EGFR and ERBB3 in monocytes. The expression of both receptors gradually increased during monocyte/PCMO culture, suggesting a role for them in the process of PCMO generation. Activation of EGFR on monocytes has been reported to be required for monocyte activation and cellular motility [17]. EGF was found also to mediate monocyte chemotaxis and macrophage proliferation [18]. Taking advantage of the relative ability of monocyte subpopulations to undergo proliferation and generate PCMOs, we showed here that EGF and HB-EGF were able to increase total cell counts and the cells' 

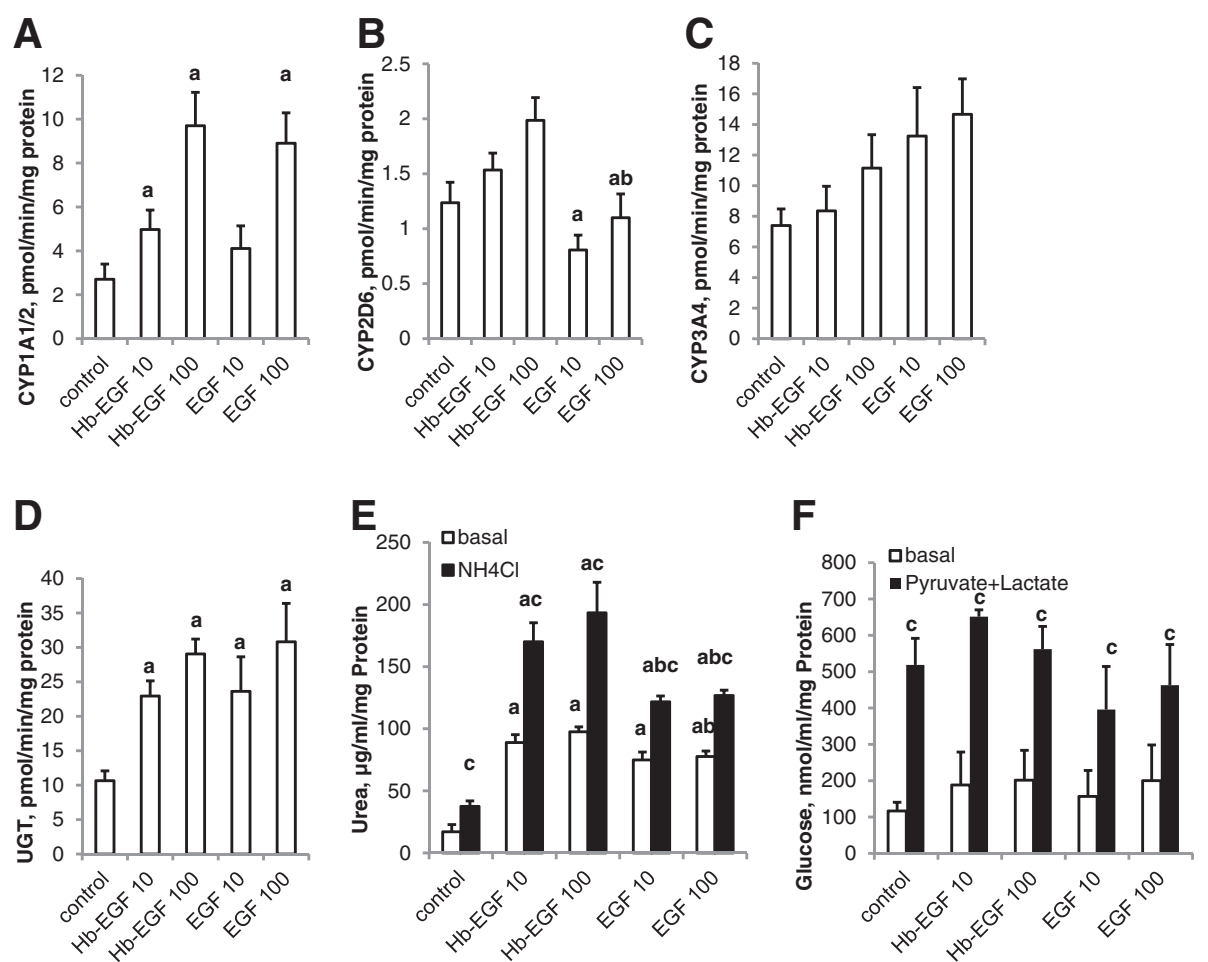

Figure 5 Effect of EGF and HB-EGF treatment during PCMO culture on NeoHepatocyte function. PCMOs treated with the indicated concentrations (in $\mu \mathrm{g} / \mathrm{L}$ ) of EGF or HB-EGF were cultured in hepatocyte conditioning medium for 2 weeks before subjecting them to analysis of cytochrome P450 (CYP) isoforms 1A1/2 (A), 2D6 (B), and 3A4 (C), the phase II enzyme UDP-glucuronosyl transferase (D), urea metabolism (E), and glucose metabolism (F). Methodologic details are given in the Methods section. Data are presented as mean \pm SEM of $N=4$. Statistical analysis: Single-Factor ANOVA: $\mathrm{p}<0.01$ for A, B and D; ANOVA (2-Factor with replication) for $\mathrm{E}: \mathrm{p}<0.001$ between different EGF/HB-EGF treatments; $\mathrm{p}<0.001$ between basal and $\mathrm{NH}_{4} \mathrm{Cl}$-treatments; for $\mathrm{F}: \mathrm{p}<0.001$ between basal and pyruvate + lactate treatments; $a=$ significantly different vs. control; $b=$ vs. corresponding HB-EGF value; $c=$ vs. basal value.

proliferative activity as assessed by Ki67 staining. With respect to Ki67 staining the HB-EGF effect did not reach statistical significance, which may be explained by donor-specific variations in the monocyte's ability to respond to various treatments in culture (H.U., unpublished observation). The enhanced proliferation was accompanied by activation of cell cycle regulatory genes ANAPC2, ABL1, CDK4, CDK6, and CDC2. ANAPC2 plays an important role in the regulation of the $\mathrm{G} 1 / \mathrm{S}$ and G2/M transitions while ABL1 regulates the S-phase and DNA replication. CDK4 and 6 participate in the G1/ $\mathrm{S}$ transition and $\mathrm{CDC} 2$ in $\mathrm{M}$-phase regulation. EGF was also previously reported to induce increased cyclin D1 expression in other systems [22]. Inhibition of some of the functional proteins such as ANAPC2 and CDC2 that form the anaphase promoting complex/cyclosome $(\mathrm{APC} / \mathrm{C})$ has been reported to induce cell cycle arrest at G2/M [23]. Thus, the induction of cell cycle arrest is associated with the down-regulation of genes involved in G1/S and G2/M transitions and an increase in the expression of these genes can lead to activation of the cell cycle. We confirmed these results by immunoblotting of $\mathrm{pRb}$, which negatively regulates progression from $\mathrm{G} 0$ through to G1 and into S phase [24]. The results showed that treatment with EGF increased the $\mathrm{pRb}$ hyperphosphorylated form to a greater extent than HB-EGF which also showed a higher degree of phosphorylation than the control. pRb is normally hypophosphorylated in resting cells at G0 when proliferation is repressed. Upon activation of the cell cycle, appropriate signals lead to the subsequent activation of the cyclin D/CDK4 and 6, cyclin E/CDK2 and cyclin A/CDK2 complexes, which increasingly phosphorylate $\mathrm{pRb}$ during progression through G1. The pRb will be kept in a hyperphosphorylated (inactive) form until late in mitosis [25].

In contrast to GM-CSF [26], M-CSF and IL-3 induced tyrosine phosphorylation and activation of ERK in monocytes. Moreover, addition of the MEK inhibitor U0126 prevented M-CSF + IL-3-induced proliferation, strongly suggesting that MEK/ERK signaling drives the proliferative response of monocytes under standard culture conditions. In the present work, we demonstrated that addition of EGF or HB-EGF superactivated the MEK/ ERK pathway and further increased proliferation. In other systems, the EGFR tyrosine kinase inhibitor Erlotinib, and U0126 completely inhibited EGF-induced 
proliferation [22]. Also, HB-EGF enhanced phosphorylation of Akt and ERK, implying a role for phosphatidylinositol 3-kinase (PI3K)/Akt and MEK/ERK signaling in HB-EGF-stimulated cell proliferation [16]. The PI3K inhibitors LY294002 and wortmannin, and the MEK/ ERK inhibitors U0126 and PD98059, reduced HB-EGFinduced BrdU incorporation into cultures [16]. Taken together, it can be concluded that exposure of PCMOs to EGF or HB-EGF leads to activation of their receptors (ERBB1 and 3), the expression of which increases during PCMO culture, and subsequent activation of MEK/ERK. This extra input of ERK signaling is sufficient to further enhance PCMO proliferation beyond the level achieved with M-CSF + IL-3-induced ERK activation.

Our results showed that both EGF and HB-EGF activated cell proliferation-associated changes in PCMOs during their generation but that these effects were generally stronger for EGF. Nevertheless, treatment with both agents resulted in the same increase in total PCMO cell numbers. This suggests the possibility that HB-EGF, in addition to its growth-promoting function, exerts anti-apoptotic effects on PCMOs that contribute to cell expansion.

Interestingly, EGF and HB-EGF appear to enhance dedifferentiation of PCMOs (as assessed by a decrease in the expression of $\mathrm{p} 47^{\text {phox }}$ ) and to increase pluripotency (as assessed by an induction of Nanog) [6]. We have previously characterized stem cell marker expression in PCMOs and have demonstrated similar expression profiles of Nanog and Oct3/4 during PCMO generation [6]. Moreover, the expression of Nanog and Oct $3 / 4$ was paralleled by a global rise in histone $\mathrm{H} 3$ methylation on Lys-4, a marker of active chromatin, and coincided with peak sensitivity to hepatocyte-specific differentiation [6].

Functionally, both EGF and HB-EGF applied during PCMO generation improved the function of the resulting NeoHepatocytes when compared with those derived from control (standard) PCMOs. However, the present results demonstrated functional similarities of NeoHepatocytes obtained after PCMO treatment with either EGF or HB-EGF. When EGF and HB-EGF where compared for their potency to enhance the hepatocyte-specific functions of NeoHepatocytes no major differences were observed, although HB-EGF appears to be superior with respect to urea production, glucose metabolism and CYP2D6 activity, while EGF was superior in inducing CYP3A4 activity.

\section{Conclusions}

The present data reveal that EGF and HB-EGF improve the proliferation of PCMOs by superactivating MEK/ ERK signaling. Notably, however, both factors improve hepatocyte-specific functions of the resulting NeoHepatocytes which is an important issue when considering these cells for transplantation purposes. Based on these data, we suggest modifying the current protocol of PCMO generation by adding EGF or HB-EGF to the culture medium.

\section{Methods \\ Generation of PCMOs}

Human peripheral blood monocytes isolated from LRS chambers or buffy coats from healthy donors were isolated by density gradient centrifugation and further purified by adherence separation. Cells $\left(1 \times 10^{5} / \mathrm{cm}^{2}\right)$ were allowed to adhere to tissue culture plastics for $1-2 \mathrm{~h}$ in RPMI 1640 medium containing 10\% human AB serum (Lonza, Cologne, Germany), $2 \mathrm{mmol} / \mathrm{L}$ glutamine, 100 $\mathrm{U} / \mathrm{mL}$ penicillin, and $100 \mu \mathrm{g} / \mathrm{mL}$ streptomycin (all from Invitrogen, Karlsruhe, Germany). Nonadherent cells were removed by aspiration, and the adherent monocytes were cultured for 4 days in dedifferentiation medium consisting of RPMI supplemented with $140 \mu \mathrm{mol} / \mathrm{L}$ 2-mercaptoethanol, $5 \mu \mathrm{g} / \mathrm{L} \mathrm{M}$-CSF, and $0.4 \mu \mathrm{g} / \mathrm{L}$ human IL-3 (both from R\&D Systems, Wiesbaden, Germany)). In previous experiments these cells have been tested for purity by flow cytometry analysis of CD45 and CD14, typically yielding a purity of $70-80 \%$ [2]. Either EGF or HB-EGF (both from R\&D Systems) was added to the dedifferentiation medium at various concentrations $(10,50$, or $100 \mu \mathrm{g} / \mathrm{L})$. The MEK inhibitor U0126 was purchased from Calbiochem/Merck (Darmstadt, Germany) and dissolved in dimethyl sulfoxide.

\section{Differentiation of PCMOs into NeoHepatocytes}

After 4 days of culture in dedifferentiation medium PCMOs were cultured for 2 weeks with hepatocyte conditioning medium (RPMI 1640 medium containing $3 \mu \mathrm{g} /$ L fibroblast growth factor-4 (FGF-4, R\&D Systems) and $10 \%$ FBS) for differentiation into NeoHepatocytes [2]. The medium was changed every 3 days. Cells were then subjected to analysis of hepatocyte function.

\section{Immunofluorescence}

PCMOs were washed with PBS, centrifuged and diluted with PBS containing 1\% BSA, centrifuged at maximal speed for 3 min using the Cytospin 4 centrifuge (Thermo Scientific) and kept in $-20^{\circ} \mathrm{C}$ until needed. For proliferative cell staining, slides were fixed in $1 \%$ paraformaldehyde, blocked for $1 \mathrm{~h}$ and then incubated with anti-human CD14 antibody (BD Biosciences, Heidelberg, Germany) at room temperature for $2 \mathrm{~h}$ and Alexafluor 488-labeled secondary antibody (Invitrogen) for $1 \mathrm{~h}$. After washing, cells were permeabilized using $0.5 \%$ triton X-100 and incubated overnight with the antihuman Ki67 (BD Pharmingen) at $4^{\circ} \mathrm{C}$ followed by Alexafluor 555-labeled secondary antibody (Invitrogen). Ki67-positive cells were counted double-blind by two 
investigators in at least 4 visual fields per slide, repeated for all experiments $(\mathrm{N}=4)$ and related to the total cell count of CD14-positive monocytes in the same field.

\section{RNA isolation and quantitative RT-PCR}

Total RNA isolation from PCMOs, human peripheral blood monocytes and autologous lymphocytes (purified by elutriation as described earlier [6]) was performed using the GeneJet purification kit (Fermentas, St. LeonRot, Germany). To assure absence of genomic DNA, all RNA samples were treated with DNase I, and primers spanning multiple exon-intron boundaries were used. For reverse transcription, $1 \mu \mathrm{g}$ of the total RNA was reverse transcribed to first strand complementary DNA using the High-Capacity reverse transcription kit (Applied Biosystems, Darmstadt, Germany). Gene expression was quantified by standard endpoint RT-PCR and standard real-time RT-PCR (qPCR) on an iCycler (BioRad, Munich, Germany) and analyzed by agarose gel electrophoresis and iCycler iQ Real-Time Detection System software (Bio-Rad), respectively. The thermal cycling program was $10 \mathrm{~min}$ at $95^{\circ} \mathrm{C}$ for enzyme activation, denaturation for $15 \mathrm{~s}$ at $95^{\circ} \mathrm{C}, 60 \mathrm{~s}$ annealing at $60^{\circ} \mathrm{C}$, and $60 \mathrm{~s}$ extension at $72^{\circ} \mathrm{C}$. A dissociation curve was performed for each product to assure the absence of primer dimers or unspecific products. Primers used in the present study are listed in Table 1 . Relative quantification was performed by $\Delta \Delta \mathrm{Ct}$ method. To normalize expression data, amplification of the housekeeping gene GAPDH was used as an internal control.

\section{Western blotting}

Following 4 days of PCMO generation, cells were thoroughly washed with PBS to remove non-adherent cells (mainly $\mathrm{T}$ lymphocytes) and lysed using PhosphoSafe lysis buffer (Novagen/Merck). Cell lysates were separated by electrophoresis prior to transfer to PVDF membranes (Immobilon P). Membranes were then probed with primary antibodies and immunoreactive bands were detected by chemiluminescence. Primary antibodies used were MEK1 (C-18), MEK2 (C-16), p-MEK1/2 (Ser218/ Ser222), ERK1 (C-16), p-ERK (Tyr 204) (all from Santa Cruz Biotechnology, Heidelberg, Germany), anti-human pRb (BD Pharmingen), and $\beta$-actin (Sigma, Deisenhofen, Germany). Secondary antibodies were obtained from GE Healthcare (Buckinghamshire, UK).

\section{Analysis of NeoHepatocyte function}

Urea measurement: To remove residual urea from the culture medium, cells were washed twice with DPBS. To determine basal levels of urea formed, cells were incubated with DPBS ( $1 \mathrm{mM} \mathrm{MgCl}_{2}, 1 \mathrm{mM} \mathrm{Na-pyruvate)} \mathrm{for}$ $24 \mathrm{~h}$. To measure the ability of the cells to metabolize ammonium, the buffer was supplemented with $5 \mathrm{mM}$
$\mathrm{NH}_{4} \mathrm{Cl} \pm 1 \mathrm{mM}$ ornithine. Supernatant $(80 \mu \mathrm{l})$ was incubated with $60 \mu \mathrm{l} \quad 0.0002 \%$ O-phthaldehyde solution $\left(0.03 \%\right.$ Brij-35, $\left.7.4 \% \mathrm{H}_{2} \mathrm{SO}_{4}\right)$ and $60 \mu \mathrm{l}$ NED reagent [0.0006\% N-(1-naphthyl)ethylenediamine dihydrochloride, $0.5 \%$ boric acid, $0.03 \%$ Brij-35, 22.2\% $\mathrm{H}_{2} \mathrm{SO}_{4}$ ] for $2 \mathrm{~h}$ at $37^{\circ} \mathrm{C}$. Absorbance was measured at $505 \mathrm{~nm}$ and compared to standard samples [21].

Glucose measurement: Cells were washed three times with DPBS before incubation for $24 \mathrm{~h}$ with DPBS $\left(30 \mathrm{mM} \mathrm{KCl}, 1 \mathrm{mM} \mathrm{MgCl}_{2}, 1 \mathrm{mM} \mathrm{Na}\right.$ pyruvate $\pm 10 \mathrm{mM}$ Na-L-lactate). Supernatant $(100 \mu \mathrm{l})$ was incubated with $150 \mu \mathrm{l}$ GLOX solution (250 mM Tris, $0.2 \mathrm{mM}$ EDTA, $0.04 \%$ glucose-oxidase, $0.007 \%$ peroxidase, $0.01 \%$ O-dianisidine, $\mathrm{pH} 8.0$ ) for $2 \mathrm{~h}$ at $37^{\circ} \mathrm{C}$. Absorbance was measured at $420 \mathrm{~nm}$ and compared to standard samples.

Phase I and II Enzyme activity assays: Fluorescencebased cytochrome P450 assays were performed by incubation of intact cells with selected substrates as reported [21]. Briefly, cells cultured on a 96-well plate were serum starved (RPMI 1640 medium without supplements) overnight prior to measurement. For measurement the medium was replaced with $100 \mu \mathrm{l}$ reaction buffer (plain RPMI 1640 medium containing the fluorogenic substrates: $25 \mu \mathrm{mol} / \mathrm{L}$ 7-ethoxy coumarin for CYP1A1/2, $10 \mu \mathrm{mol} / \mathrm{L}$ AMMC (3-[2-(N,N-diethyl-N-methylamino)ethyl]-7-methoxy-4methylcoumarin) for CYP2D6, $10 \mu \mathrm{mol} / \mathrm{L}$ BFC (7benzyloxy-4-trifluoromethylcoumarin) for CYP3A4 and $100 \mu \mathrm{mol} / \mathrm{L}$ 4-methylumbelliferon as a substrate for UDPGlucuronosyl-transferase). Fluorescence was measured every 10 min over a period of $2 \mathrm{~h}$ with a microplate reader (BMG Labtech, Offenburg, Germany). Afterwards cells were fixed for protein quantification by sulforhodamine B (SRB) staining as previously described [27]. Results are given as pmol of fluorescent product formed (phase I enzyme activities) or fluorescent substrate reduced (for phase II enzyme activities) per minute normalized to total protein content in $\mathrm{mg}$.

\section{Statistical analysis}

All samples were measured in duplicates. Values were expressed as mean \pm SEM. with $\mathrm{N}=4$ in all experiments. Group statistical comparisons were performed by oneway or two-way analysis of variances (ANOVA) followed by Mann-Whitney multi-range analysis as a post-hoc test. The $p$ values were shown in the Results section A statistical difference was considered significant if $p<0.05$.

\section{Abbreviations}

CDK: Cyclin-dependent kinase; EGF: Epidermal growth factor:

ERK: Extracellular signal-regulated kinase; FBS: Fetal bovine serum; GM-CSF: Granulocyte macrophage colony-stimulating factor; h: Hour; HB-EGF: Heparin-binding epidermal growth factor; IL-3: Interleukin-3; M-CSF: Macrophage colony stimulating factor; PBS: Phosphate-buffered saline; PCMOs: Programmable cell(s) of monocytic origin; 
pRb: Retinoblastoma protein; s: Second; SDS-PAGE: Sodium dodecyl sulfate polyacrylamide gel electrophoresis.

\section{Competing interests}

The authors have no competing interests to declare.

\section{Authors' contributions}

$\mathrm{AH}, \mathrm{FF}$, and $\mathrm{HU}$ designed the study and drafted the manuscript; $\mathrm{AH}, \mathrm{SE}$, and AN coordinated and conducted the experiments. All authors read and approved the final manuscript.

\section{Acknowledgements}

We thank Dr. N. Reiling (Research Center Borstel, Borstel, Germany) for the kind donation of highly pure preparations of monocytes and autologous lymphocytes. This work was supported in part by a grant from the "Bundesministerium für Bildung und Forschung" (01 GN 0985).

\section{Author details}

${ }^{1}$ Clinic for Applied Cellular Medicine, UKSH, Campus Kiel, Arnold-Heller Strasse 3, Hs. 18, 24105 Kiel, Germany. ${ }^{2}$ BG Unfallklinik Tübingen, Eberhard-Karls Universität Tübingen, Schnarrenbergstr. 95, 72076 Tübingen, Germany. ${ }^{3}$ First Department of Medicine, UKSH, Campus Lübeck, Ratzeburger Allee 160, 23538 Lübeck, Germany.

Received: 9 March 2012 Accepted: 22 June 2012

Published: 8 August 2012

\section{References}

1. Hengstler JG, Brulport M, Schormann W, Bauer A, Hermes M, Nussler AK, Fandrich F, Ruhnke M, Ungefroren $\mathrm{H}$, Griffin L, Bockamp E, Oesch F, von Mach MA: Generation of human hepatocytes by stem cell technology: definition of the hepatocyte. Expert Opin Drug Metab Toxicol 2005, 1:61-74.

2. Ruhnke M, Ungefroren $H$, Nussler A, Martin F, Brulport M, Schormann W, Hengstler J, Klapper W, Ulrichs K, Hutchinson J, Soria B, Parwarsch R, Heeckt $P$, Kremer B, Faendrich F: Differentiation of in vitro-modified human peripheral blood monocytes into hepatocyte-like and pancreatic isletlike cells. Gastroenterology 2005, 128:1774-1786.

3. Ruhnke M, Nussler AK, Ungefroren H, Hengstler JG, Kremer B, Hoeckh W, Gottwald T, Heeckt P, Fandrich F: Human monocyte-derived neohepatocytes: a promising alternative to primary human hepatocytes for autologous cell therapy. Transplantation 2005, 79:1097-1103.

4. Seta N, Kuwana M: Derivation of multipotent progenitors from human circulating CD14+ monocytes. Exp Hematol 2010, 38:557-563.

5. Zhao Y, Glensne D, Huberman E: A human peripheral blood monocytederived subset acts as pluripotent stem cells. Proc Nat Acad Sci USA 2003, 100:2426-2431.

6. Ungefroren H, Groth S, Hyder A, Thomsen N, Hinz H, Reiling N, GrageGriebenow E, Held-Feindt J, Schulze M, Nüssler AK, Fändrich F: The generation of programmable cells of monocytic origin involves partial repression of monocyte/macrophage markers and reactivation of pluripotency genes. Stem Cells Dev 2010, 19:1769-1780.

7. Clanchy FIL, Holloway AC, Lari R, Cameron PU, Hamilton JA: Detection and properties of the human proliferative monocyte subpopulation. I Leukoc Biol 2006, 79:757-766.

8. Hoelting T, Siperstein AE, Clark OH, Duh QY: Epidermal growth factor enhances proliferation, migration, and invasion of follicular and papillary thyroid cancer in vitro and in vivo. J Clin Endocrinol Metab 1994, 79:401-408.

9. Li T, Lu L: Epidermal growth factor-induced proliferation requires down-regulation of Pax6 in corneal epithelial cells. J Biol Chem 2005, 280:12988-12995.

10. Rescan C, Le Bras S, Lefebvre V, Frandsen U, Klein T, Foschi M, Pipeleers D, Scharfmann R, Madsen O, Heimberg H: EGF-induced proliferation of adult human pancreatic duct cells is mediated by the MEK/ERK cascade. Lab Invest 2005, 85:65-74.

11. Pennock S, Wang Z: Stimulation of cell proliferation by endosomal epidermal growth factor receptor as revealed through two distinct phases of signaling. Mol Cell Biol 2003, 23:5803-5815.

12. Schiff B, McMurphy A, Jasser S, Younes M, Doan D, Yigitbasi O, Kim S, Zhou G, Mandal M, Bekele B, Holsinger F, Sherman S, Yeung S, El-Naggar A, Myers $\mathrm{J}$ : Epidermal growth factor receptor (EGFR) is overexpressed in anaplastic thyroid cancer, and the EGFR inhibitor Gefitinib inhibits the growth of anaplastic thyroid cancer. Clin Cancer Res 2004, 10:8594-8602.

13. Higashiyama S, Lau K, Besner GE, Abraham JA, Klagsbrun M: Structure of heparin-binding EGF-like growth factor. Multiple forms, primary structure, and glycosylation of the mature protein. J Biol Chem 1992, 267:6205-6212.

14. Hashimoto K, Higashiyama S, Asada H, Hashimura E, Kobayashi T, Sudo K, Nakagawa T, Damm D, Yoshikawa K, Taniguchi N: Heparin-binding epidermal growth factor-like growth factor is an autocrine growth factor for human keratinocytes. J Biol Chem 1994, 269:20060-20066.

15. Nolan T, Girolamo N, Goroneo M, Wakefield D: Proliferative effects of heparin-binding epidermal growth factor-like growth factor on pterygium epithelial cells and fibroblasts. Investig Opthahlmol Vis Sci 2004, 45:110-113.

16. Jin K, Mao XO, Del Rio Guerra G, Jin L, Greenberg DA: Heparin-binding epidermal growth factor-like growth factor stimulates cell proliferation in cerebral cortical cultures through phosphatidylinositol 3'-kinase and mitogen-activated protein kinase. J Neurosci Res 2005, 81:497-505.

17. Chan G, Nogalski MT, Yurochko AD: Activation of EGFR on monocytes is required for human cytomegalovirus entry and mediates cellular motility. Proc Natl Acad Sci USA 2009, 106:22369-22374.

18. Lamb DJ, Modjtahedi H, Plant NJ, Ferns G: EGF mediates monocyte chemotaxis and macrophage proliferation and EGF receptor is expressed in atherosclerotic plaques. Atherosclerosis 2004, 176:21-26.

19. Vietri M, Bianchi M, Ludlow JW, Mittnacht S, Villa-Moruzzi E: Direct interaction between the catalytic subunit of protein phosphatase 1 and pRb. Cancer Cell Int 2006, 6:3-11.

20. Pagano M, Pepperkok R, Verde F, Ansorge W, Draetta G: Cyclin A is required at two points in the human cell cycle. EMBO J 1992, 11:961-971.

21. Ehnert S, Seeliger C, Vester H, Schmitt A, Saidy-Rad S, Lin J, Neumaier M, Gillen S, Kleeff J, Friess H, Burkhart, Stöckle U, Nüssler AK: Autologous serum improves yield and metabolic capacity of monocyte-derived hepatocytelike cells: Possible implication for cell transplantation. Cell Transplant 2011, 20:1465-1477.

22. Lee J, Ryu SH, Kang SM, Chung WC, Gold KA, Kim ES, Hittelman WN, Ki Hong W, Koo JS: Prevention of bronchial hyperplasia by EGFR pathway inhibitors in an organotypic culture model. Cancer Prev Res (Phila) 2011, 4:1306-1315.

23. Heilman DW, Green MR, Teodoro JG: The anaphase promoting complex: a critical target for viral proteins and anticancer drugs. Cell Cycle 2005, 4:560-563.

24. Sidle A, Palaty C, Dirks P, Wiggan O, Kiess M, Gill RM, Wong AK, Hamel PA: Activity of the retinoblastoma family proteins, pRB, p107, and p130, during cellular proliferation and differentiation. Crit Rev Biochem Mol Biol 1996, 31:237-271.

25. De Falco G, Comes F, Simone C: pRb: master of differentiation. Coupling irreversible cell cycle withdrawal with induction of muscle-specific transcription. Oncogene 2006, 25:5244-5249.

26. Yagisawa M, Saeki K, Okuma E, Kitamura T, Kitagawa S, Hirai H, Yazaki Y, Takaku F, Yuo A: Signal transduction pathways in normal human monocytes stimulated by cytokines and mediators: comparative study with normal human neutrophils or transformed cells and the putative roles in functionality and cell biology. Exp Hematol 1999, 27:1063-1076.

27. Ehnert S, Nussler AK, Lehmann A, Dooley S: Blood monocyte-derived NeoHepatocytes as in vitro test system for drug metabolism. Drug Metab Dispos 2008, 36:1922-1929.

\section{doi:10.1186/1478-811X-10-23}

Cite this article as: Hyder et al: EGF and HB-EGF enhance the proliferation of programmable cells of monocytic origin (PCMO) through activation of MEK/ERK signaling and improve differentiation of PCMO-derived hepatocyte-like cells. Cell Communication and Signaling 2012 10:23. 